\title{
Naturally Occurring Helicobacter pylori Infection in the Cynomolgus Monkey (Macaca fascicularis) Used in Subclinical Studies
}

\author{
Osamu Katsuta ${ }^{1}$, Yuki Tomonari ${ }^{1}$, Yoshimasa Okazaki ${ }^{1}$, Makoto Kawamura ${ }^{2}$, \\ Kazuo Watanabe ${ }^{2}$, Hiroyuki Matsui ${ }^{2}$, Yoshihiro Sakuma ${ }^{1}$, and Minoru Tsuchitani ${ }^{1}$ \\ ${ }^{1}$ Mitsubishi Chemical Safety Institute Ltd., Ibaraki-ken 314-0255, Japan \\ ${ }^{2}$ Kazusa Research Center, Mitsubishi-Tokyo Pharmaceuticals Inc., Chiba-ken 292-0812, Japan
}

\begin{abstract}
Recently, a high incidence of naturally occurring Helicobacter pylori (H. pylori)-related gastritis was reported in a cynomolgus monkey colony (Reindel et al., 1999). We retrospectively examined 82 cynomolgus monkeys from three different countries. These monkeys were used in toxicological or pharmaceutical studies in our laboratory. Diffuse antral gastritis was observed in 49 of 58 (84\%) animals of Group A in the Philippines, and two of 10 (20\%) of Group $\mathrm{C}$ in Japan. In their antrum, the mucosal layer was thickened with lymphocytic and plasmacytic infiltration, and enlarged lymphoid follicles. However, no erosion or hemorrhage was seen in the lesions. H. pylori-like bacteria were detected by Warthin-Starry staining in almost all of the monkeys showing antral gastritis. No histological changes or H. pylori-like bacteria were detected in the animals of Group B in Indonesia. In conclusion, although the incidence of infection may be in part due to the breeder, natural infection of $H$. pylori was confirmed in cynomolgus monkeys. Thus, this species as well as rhesus monkeys may be useful in the study of $H$. pylori infection. Moreover, because of its high incidence, the colony in the Philippines may be suitable for an animal model, as it is comparable with natural infection in humans. (J Toxicol Pathol 2001; 14: 45-49)
\end{abstract}

Key words: Helicobacter pylori infection, cynomolgus monkeys, antral gastritis

Helicobacter pylori (H. pylori) is a serious pathogen in humans causing chronic atrophic gastritis and persistent ulcer, and is related to some gastric tumors ${ }^{1}$. The same gastric changes as those in humans have been reported in non-human primates by natural ${ }^{2-7}$ or experimental ${ }^{2,8-13}$ infection with this pathogen. There have been many studies of $H$. pylori infection in rhesus monkeys (Macaca mulatta) $)^{2-7}$, 12,13 , and this monkey species is regarded as a good human model for infection with this pathogen. Despite belonging to the same genus, Macacus, cynomolgus monkeys (Macaca fascicularis) are less susceptible to $H$. pylori infection than rhesus monkeys ${ }^{2}$. Therefore, there have been few reports concerning infection of cynomolgus monkeys with this pathogen $^{2,10}$.

Recently, a high incidence of naturally occurring $H$. pylori-related gastritis was reported in a cynomolgus monkey colony of a pharmaceutical research laboratory ${ }^{14}$. According to the article, the monkeys originated from the

Received: 24 October 2000, Accepted: 7 January 2001

Mailing address: Osamu Katsuta, Mitsubishi Chemical Safety Institute Ltd., 14 Sunayama, Hasaki-machi, Kashima-gun, Ibaraki-ken 3140255, Japan

TEL: 81-479-46-2871 FAX: 81-479-46-2874

E-mail: okatsuta@ankaken.co.jp
Philippines. We retrospectively examined formalin-fixed stomach specimens of 82 cynomolgus monkeys, which were used and sacrificed in toxicological or pharmaceutical studies from 1995 to 1998 (Table 1) in our laboratory. The test substances had no effects on the stomach. The monkeys were obtained from commercial breeders in three different countries; 58 animals (Group A) from the Philippines (Simian Conservation Breeding \& Research Center, Inc.), 14 animals (Group B) from Indonesia (C. V. Primates, Inc.), and 10 animals (Group C) from Japan (Japan E. D. M., Inc.). Group A in the Philippines and Group C in Japan adopted a colony-breeding system, and the monkeys of Group A may have come from the same colony as that of a previous study ${ }^{14}$. The monkeys of Group B were captured and raised in Indonesia.

Two specimens of the gastric fundus and antrum from each animal were stained with hematoxylin and eosin (HE), and Warthin-Starry stain. Selected specimens were immunohistochemically stained with a polyclonal rabbit anti-H. pylori antibody (Signet, Dedham, MA, USA) using the labeled streptavidin-biotin method (Dako LSAB Kit; Dako Co., Carpinteria, CA, USA). Selected samples from the gastric mucosa of the fundus and antrum were taken from the formalin-fixed tissue for transmission electron 
Table 1. Histopathological Findings of the Stomach in Cynomolgus Monkeys

\begin{tabular}{lccc}
\hline & Group A & Group B & Group C \\
\hline Number of monkeys examined (male, female) & $58(30,28)$ & $14(7,7)$ & $10(-, 10)$ \\
Mean age (range) & $3.8(3-5)$ & $7.6(5-13)$ & $5.2(5-6)$ \\
\hline & M\&F (M, F)\# & M\&F (M, F) & M\&F (M, F) \\
Diffuse antral gastritis & $49(25,24)$ & 0 & $2(-, 2)$ \\
Lymphoplasmacytic infiltration & $50(26,24)$ & 0 & $2(-, 2)$ \\
Mucosal hyperplasia & $46(22,24)$ & 0 & $2(-, 2)$ \\
Enlarged lymph follicle & $43(22,21)$ & $1(1,0)$ & $1(-, 1)$ \\
Microabscess & $23(9,14)$ & 0 & 0 \\
Fundic gastritis & $12(6,6)$ & 0 & 0 \\
$-------\ldots$ - - - - - - - - - - - - & & & 0 \\
Bacteria detected by Warthin-Starry staining & $51(27,24)$ & 0 & $2(-, 2)$ \\
H. pylori-like in the antrum & $54(27,27)$ & $14(7,7)$ & $10(-, 10)$ \\
H. heilmannii-like in the fundus & & & \\
\hline
\end{tabular}

\#M\&F (M, F), Total number of males and females (Males, Females).
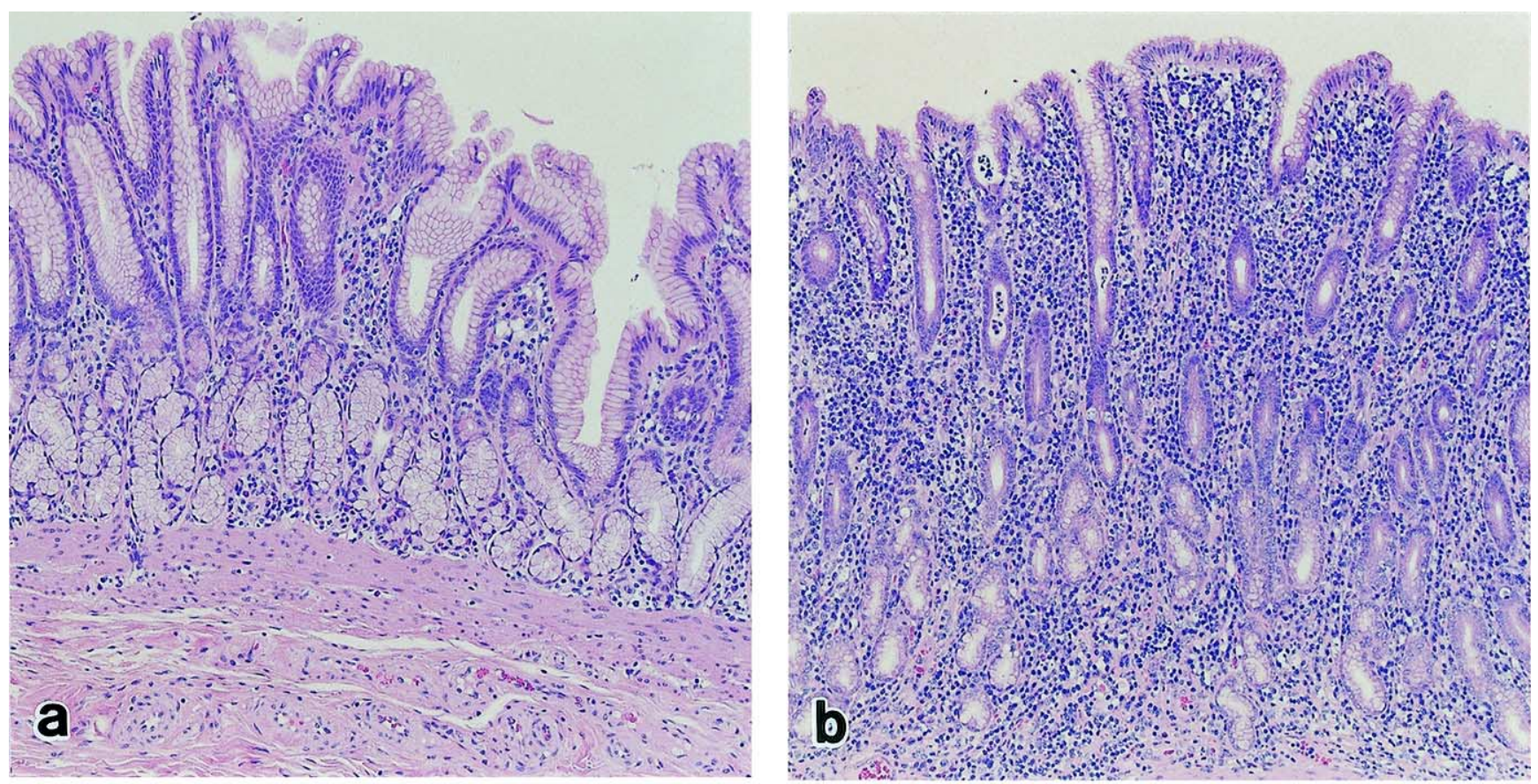

Fig. 1 Antral mucosa of cynomolgus monkeys. a: No particular change is observed in a monkey of Group B. b: Thickening of the mucosa due to marked lympho-plasmacytic infiltration. HE staining, $\times 100$.

microscopy.

No macroscopic evidence of disease was observed in any of the 82 monkeys. Diffuse antral gastritis was observed in 49 of $58(84 \%)$ animals regardless of gender, and two of $10(20 \%)$ animals in Groups A and C, respectively. In the antrum of these animals, numerous lymphocytes and plasma cells, some of which changed to Mott cells, were infiltrated into the lamina propria and into the epithelial layer of the mucosa (Figs. 1b and 2). Microabscesses were occasionally observed within the upper antral pits and glands. However, no mucosal erosion or hemorrhage was seen. Enlarged lymphoid follicles with a prominent germinal center were formed throughout the lamina propria and in the submucosal layer. Although the mucosa was thickened and its surface, pits, and glandular epithelium were hyperplastic. Furthermore, the epithelial cell height was diminished due to a decrease in the mucus content. Some bacterial clumps were visible within the upper pits and glands (Fig. 2), but the disseminated organisms could not be readily detected by HE staining. In 12 of 58 monkeys of Group A, such gastritis extended to the fundic area. However, other monkeys in both the other groups showed less marked alterations in the fundus. No changes were detected in Group B. WarthinStarry staining revealed two morphologically distinct bacteria in the antral (Fig. 3a) and fundic (Fig. 4a) mucosa. Moreover, both organisms were immunohistochemically 


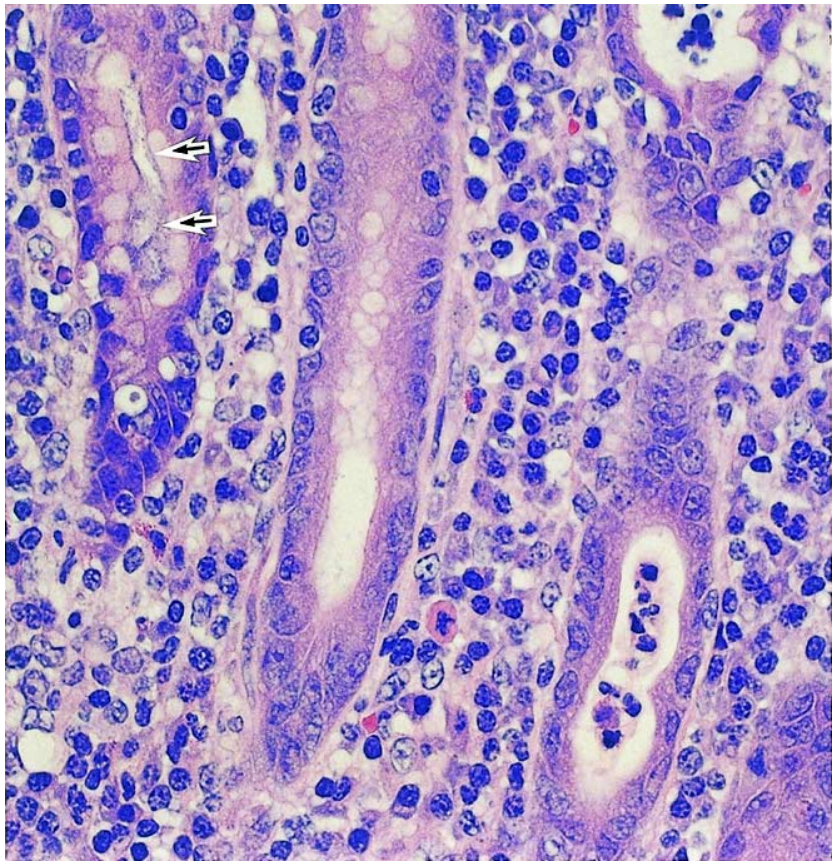

Fig. 2 Higher magnification of Fig. 1b. A bacterial clump can be seen within the upper pit (arrows). HE staining, $\times 400$.

positive with the anti-H.pylori antibody (Figs. 3b, and 4b). Concurrent infection with $H$. pylori and $H$. heilmannii-like organisms has been reported ${ }^{5,6,14}$. H. heilmannii can not be cultured in vitro and the organism can only be detected by morphological examination ${ }^{14}$. Although its pathogenicity has been recently reported in humans ${ }^{15-17}$, it has not been clear in non-human primates ${ }^{5,6,14}$. In this study, these two bacteria could be distinguished from each other based on their morphology and location, as previously reported in cynomolgus $^{14}$ and rhesus monkeys ${ }^{5,6}$. H. pylori-like bacteria, which were short in length, curved, and rod-shaped, were located in the gastric pits and the upper gastric glands of the antral mucosa. The monkeys, which were positive for H. pylori-like bacteria on Warthin-Starry staining, mostly corresponded with those showing antral gastritis. $H$. heilmannii-like bacteria, which were long, tightly coiled and spiral in shape, were found within the lumen of the pits, glands, and surface mucus of the fundic mucosa. The organisms were especially prevalent in the cytoplasm of parietal cells. These organisms were detected in almost all monkeys in this study. However, no pathological changes were observed and the prevalence of these organisms was deemed to have no relation to the antral gastritis. Ultrastructural examination also showed two different spiral bacteria. H. pylori-like bacteria with no coiling, measuring approximately $3 \mu \mathrm{m} \times 1.3 \mu \mathrm{m}$, were found in the lumen of the antral pits (Fig. 5a). H. heilmannii-like bacteria were present in the fundic glands and in the parietal cells, and had four to eight spirals measuring $8 \mu \mathrm{m} \times 1 \mu \mathrm{m}$ at maximum length (Fig. 5b). Many flagellae were seen around both organisms, but in this study, we could not confirm their polarity as previously reported ${ }^{1,4}$.
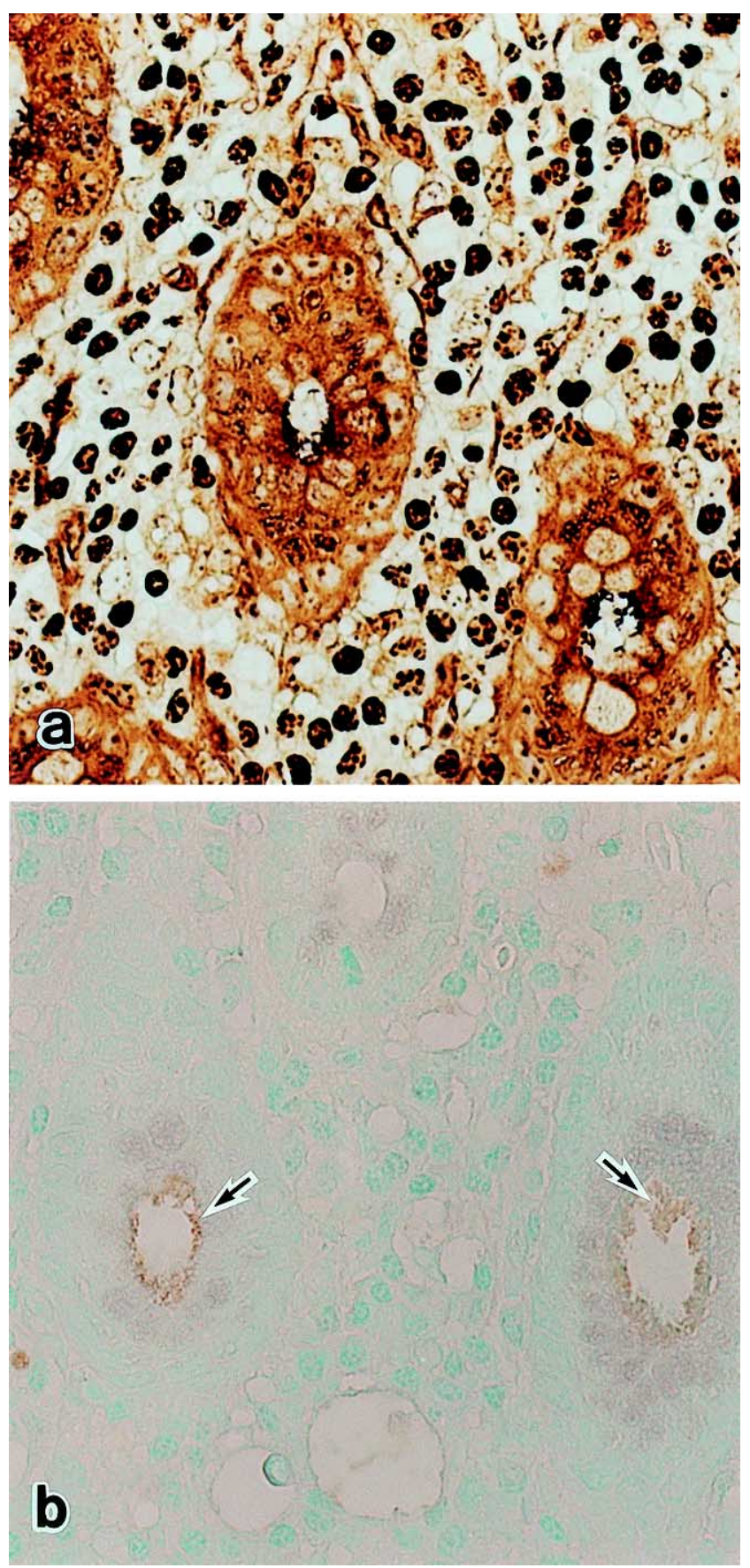

Fig. 3 Helicobacter pylori-like organisms (arrows) on the gastric gland surface. a: Warthin-Starry staining. b: AntiHelicobacter pylori antibody immunostaining counterstained with methyl green. $\times 600$.

In addition, five cynomolgus monkeys underwent gastroduodenal endoscopic examination. They were simultaneously obtained from the same colony as Group A and maintained without any treatment for two years in our laboratory. The superficial mucus and mucosal samples taken from the pyloric antrum with endoscopy were smeared onto modified Belo-Horizonte medium (M-BHM; Nikken Bio Medical Laboratory Co., Ltd., Kyoto, Japan) and cultured for five days at $35^{\circ} \mathrm{C}$ in a microaerophilic 


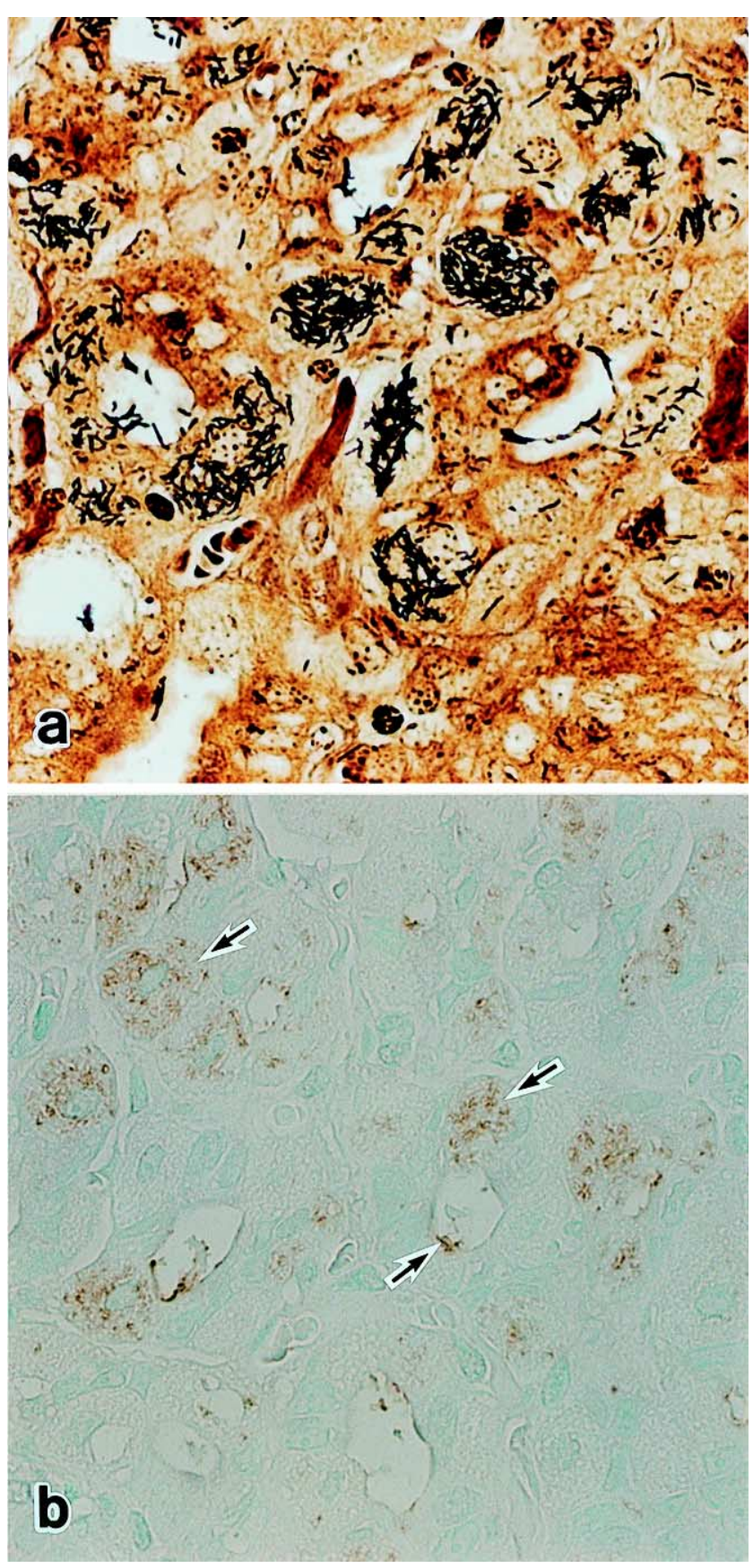

Fig. 4 Helicobacter heilmannii-like organisms (arrows) within the lumen and in the parietal cells of the fundic glands. a: Warthin-Starry staining. b: Anti-Helicobacter pylori antibody immunostaining counterstained with methyl green. $\times 600$.

atmosphere. H. pylori-like organisms were detected in three of the five monkeys, and the organisms in these animals were positive for urease, catalase, and oxidase activities. Furthermore, the isolated organisms were sensitive to both amoxicillin (minimum inhibitory concentration $(\mathrm{MIC})=$ $0.025-0.05 \mu \mathrm{g} / \mathrm{mL}$ ) and clarithromycin $(\mathrm{MIC}=0.025 \mu \mathrm{g} /$ $\mathrm{mL})$. These microbiological findings were consistent with the results in previous reports ${ }^{2,10}$. So it was strongly indicated that the organisms were $H$. pylori.
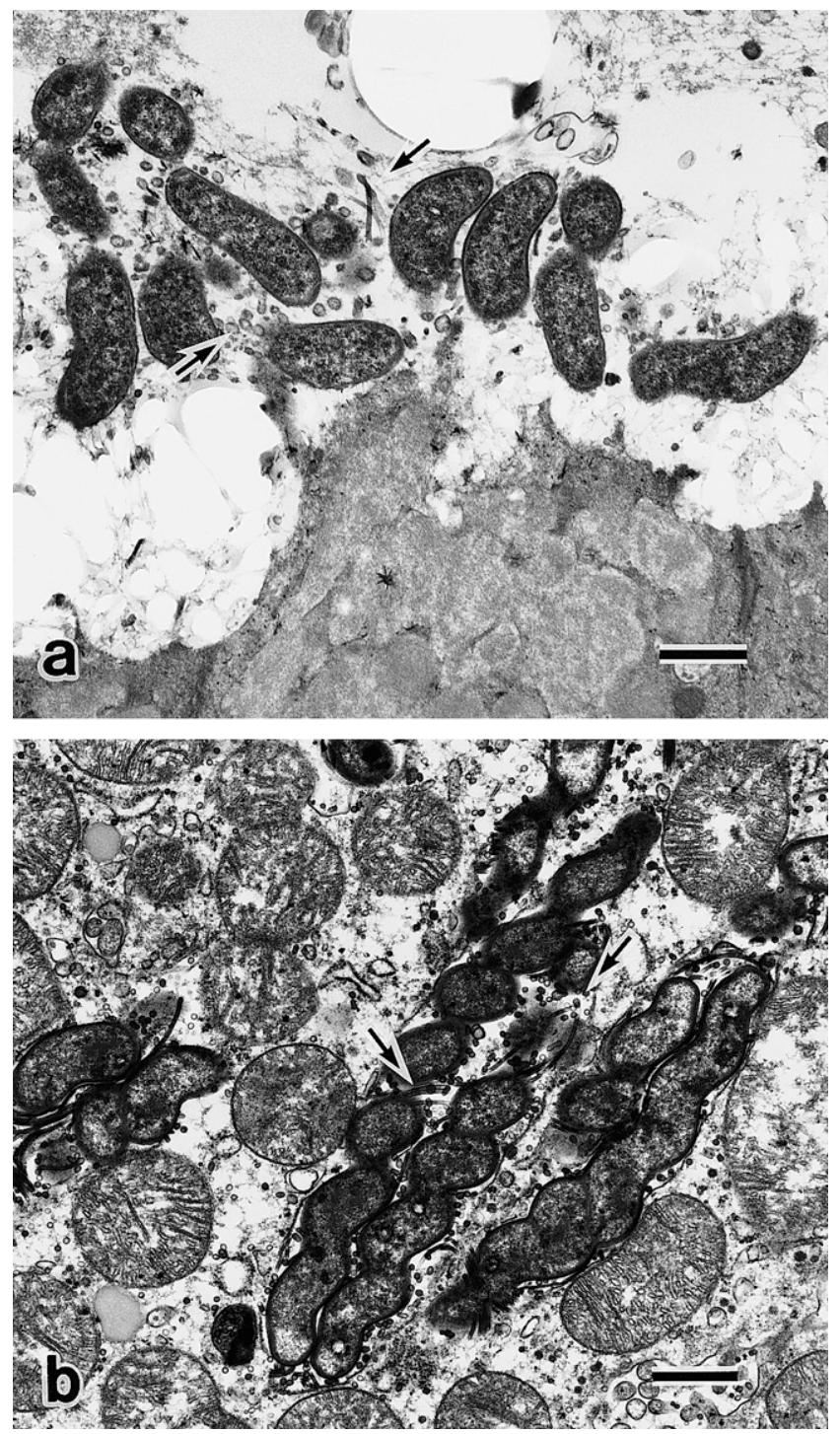

Fig. 5 Electron microscopy. a: Helicobacter pylori-like organisms with no coil. b: Helicobacter heilmannii-like organisms with tight spirals. Many flagellae are seen around both organisms (arrows). Bar $=1 \mu \mathrm{m}$

The results of this study indicated that the cynomolgus monkeys of Group A were highly infected with H. pylori. Group A was from the Philippines, and possibly the same colony as that in a previous study ${ }^{14}$. If this was the case, we may be able to reproduce and confirm their results. In addition, this study demonstrated natural infection with $H$. pylori in another country. It is, therefore, unlikely that cynomolgus monkeys are less susceptible to $H$. pylori infection than rhesus monkeys. The apparent higher susceptibility of rhesus monkeys may simply be because they are more commonly used in biomedical research than cynomolgus monkeys ${ }^{14}$.

H. pylori infection is common in captive rhesus monkeys and natural transmission is by fecal-oral and oraloral routes, and by close contact during breast feeding ${ }^{13}$. Such transmission can readily occur in colony-bred 
monkeys, even cynomolgus monkeys. Furthermore, as human-to-human transmission of the organism via endoscopy has been reported ${ }^{18}$, inadequate sterilization of gastric gavage tubes is likely to be a significant route of transmission in oral administration studies. In addition, this and the previous ${ }^{14}$ studies revealed that the colony in the Philippines had a higher incidence of $H$. pylori infection. The natural infection in the colony might be similar to the epidemiological prevalence in humans. So clinical and laboratory surveys of the monkeys in their life span are important to study $H$. pylori-infectious diseases.

Acknowledgment: We thank Mr. Steve Yamakami for language editing.

\section{References}

1. Fox JG, and Lee A. The role of Helicobacter species in newly recognized gastrointestinal tract diseases of animals. Lab Anim Sci 1997; 47: 222-255.

2. Euler AR, Zurenko GE, Moe JB, Ulrich RG, and Yagi Y. Evaluation of two monkey species (Macaca mulatta and Macaca fascicularis) as possible models for human Helicobacter pylori disease. J Clin Microbiol 1990; 28: 2285-2290.

3. Reed KD, and Berridge BR. Campylobacter-like organisms in the gastric mucosa of rhesus monkeys. Lab Anim Sci 1988; 38: 329-331.

4. Baskerville A, and Newell DG. Naturally occurring chronic gastritis and $C$ pylori infection in the rhesus monkey: a potential model for gastritis in man. Gut 1988; 29: 465-472.

5. Dubois A, Tarnawski A, Newell DG, Fiala N, Dabros W, Stachura J, Krivan H, and Heman-Ackah LM. Gastric injury and invasion of parietal cells by spiral bacteria in rhesus monkeys. Gastroenterology 1991; 100: 884-891.

6. Dubois A, Fiala N, Heman-Ackah LM, Drazek ES, Tarnawski A, Fishbein WN, Perez-Perez GI, and Blaser MJ. Natural gastric infection with Helicobacter pylori in monkeys: a model for spiral bacteria infection in humans. Gastroenterology 1994; 106: 1405-1417.

7. Drazek ES, Dubois A, and Holmes RK. Characterization and presumptive identification of Helicobacter pylori isolates from rhesus monkeys. J Clin Microbiol 1994; 32: 17991804.

8. Hazell SL, Eichberg JW, Lee DR, Alpert L, Evans DG, Evans DJ, Jr., and Graham DY. Selection of the chimpanzee over the baboon as a model for Helicobacter pylori infection. Gastroenterology 1992; 103: 848-854.

9. Shuto R, Fujioka T, Kubota T, and Nasu M. Experimental gastritis induced by Helicobacter pylori in Japanese monkeys. Infect Immun 1993; 61: 933-939.

10. Masubuchi N, Takahashi S, Nakano M, Igarashi H, Saito S, Aoyagi T, Itoh T, and Hirata I. Experimental infection of the cynomolgus monkey with Helicobacter pylori. Nippon Rinsho 1993; 51: 3127-3131.

11. Stadtländer CTKH, Gangemi JD, Stutzenberger FJ, Lawson JW, Lawson BR, Khanolkar SS, Elliott-Raynor KE, Farris HE, Jr., Fulton LK, Hill JE, Huntington FK, Lee CK, and Monath TP. Experimentally induced infection with Helicobacter pylori in squirrel monkeys (Saimiri spp.): clinical, microbiological, and histopathologic findings. Lab Anim Sci 1998; 48: 303-309.

12. Dubois A, Berg DE, Incecik ET, Fiala N, Heman-Ackah LM, Valle JD, Yang M, Wirth HP, Perez-Perez GI, and Blaser MJ. Host specificity of Helicobacter pylori strains and host responses in experimentally challenged nonhuman primates. Gastroenterology 1999; 116: 90-96.

13. Solnick JV, Canfield DR, Yang S, and Parsonnet J. Rhesus monkey (Macaca mulatta) model of Helicobacter pylori: noninvasive detection and derivation of specific-pathogenfree monkeys. Lab Anim Sci 1999; 49: 197-201.

14. Reindel JF, Fitzgerald AL, Breider MA, Gough AW, Yan C, Mysore JV, and Dubois A. An epizootic of lymphoplasmacytic gastritis attributed to Helicobacter pylori infection in cynomolgus monkeys (Macaca fascicularis). Vet Pathol 1999; 36: 1-13.

15. Mention K, Michaud L, Guimber D, Martin De Lasalle E, Vincent P, Turck D, and Gottrand F. Characteristics and prevalence of Helicobacter heilmannii infection in children undergoing upper gastrointestinal endoscopy. J Pediatr Gastroenterol Nutr 1999; 29: 533-539.

16. Morgner A, Lehn N, Andersen LP, Thiede C, Bennedsen M, Trebesius K, Neubauer B, Neubauer A, Stolte M, and Bayerdörffer E. Helicobacter heilmannii-associated primary gastric low-grade MALT lymphoma: complete remission after curing the infection. Gastroenterology 2000; 118: 821-828.

17. Svec A, Kordas P, Pavlis Z, and Novotny J. High prevalence of Helicobacter heilmannii-associated gastritis in a small, predominantly rural area: further evidence in support of a zoonosis? Scand J Gastroenterol 2000; 35: 925-928.

18. Langenberg W, Rauws EAJ, Oudbier JH, and Tytgat GNJ. Patiet-to-patient transmission of Campylobacter pylori infection by fiberoptic gastroduodenoscopy and biopsy. J Infect Dis 1990; 161: 507-511. 